\title{
Study on Pharmacokinetics and Bioequivalence of Cefdinir Dispersible Tablet in Healthy Chinese Volunteers
}

\author{
Cai-Li Zhang, Jian-Jie Jiao,Yan-Na Wu, Jun-qiu Song,Wei-Zhen Gao, De-Lu Ma and Jian-Shi Lou*
}

Division of Clinical Pharmacology, Department of Pharmacology, Tianjin Medical University, Tianjin 300070, P.R.China

\begin{abstract}
Aim: To evaluate the pharmacokinetics and bioequivalence of cefdinir dispersible tablet with cefdinir capsule in healthy Chinese volunteers.

Methods: cefdinir dispersible tablet and cefdinir capsule were used as the test and reference preparations respectively. Two randomized, comparative two-way crossover studies were conducted in 18 healthy Chinese male volunteers. Test preparation $200 \mathrm{mg}$, reference preparation $200 \mathrm{mg}$ were administered once orally to the volunteers fasting overnight, with 5 days washout period between the doses of the test and reference preparations. Before dosing blood sample, after dosing several blood samples were collected for a period of 12 hours. The plasma concentrations of cefdinir at different times were determined by high performance liquid chromatogrphy (HPLC) with ultraviolet (UV) assay. Pharmacokinetic parameters were calculated. Safety of the drug was evaluated.

Results: The main pharmacokinetic parameters of the test and reference preparations were as follows: Cmax were $1.52 \pm 0.48 \mu \mathrm{g} / \mathrm{ml}$ and $1.42 \pm 0.39 \mu \mathrm{g} / \mathrm{ml}, \mathrm{T}_{\text {mer }} 3.08 \pm 0.73 \mathrm{~h}$ and $3.22 \pm 0.81 \mathrm{~h}, \mathrm{t}_{1 / 2}$ were $2.04 \pm 0.53 \mathrm{~h}$ and $1.87 \pm 0.29 \mathrm{~h}$ AUC $_{0-\mathrm{t}}$ were $7.12 \pm 1.85 \mu \mathrm{g} / \mathrm{ml}^{\circ} \mathrm{h}^{-1}$ and $6.86 \pm 1.60 \mu \mathrm{g} / \mathrm{ml}^{\circ} \mathrm{h}^{-1} \mathrm{AUC}_{0-\infty}$ were $7.67 \pm 2.01 \mu \mathrm{g} / \mathrm{ml}^{\circ} \mathrm{h}^{-1}$ and $7.38 \pm 1.85 \mu \mathrm{g} / \mathrm{ml}^{-\mathrm{h}^{-1}}$ respectively. The relative bioavailability of test preparation was $103.53 \pm 11.50 \%$. No significant differences between two preparations were found. The parameter mean values of the pharmacokinetic characteristics for test drug were within the bioequivalence acceptable range of $80 \sim 125 \%$ and $70 \sim 143 \%$ respectively for $A U C$ and $C_{\max }$
\end{abstract}

Conclusion: The results indicate that the test preparation is bioequivalent to the reference preparation.

Keywords: Bioequivalence; Pharmacokinetics; HPLC; Cefdinir; Dispersible tablet

\section{Introduction}

Cefdinir is one of the third generation cephalosporins which have broad spectrum antibacterial activity. Cefdinir has activity against Gram-positive and Gram-negative bacteria. It is also effective against $\beta$-lactamase producing strains of haemophilis and neisseria. This drug is used to treat wide variety of sensitive bacteria infections, especially for mild and moderate infections [1]. To reduce the development of drug-resistant bacteria and maintain the effectiveness of cefdinir, it should be used only to treat or prevent infections that are proven or strongly suspected to be caused bacteria. Cefdinir capsule, tablet and suspension are available in the market for several years [2]. Now, cefdinir dispersible tablet is investegated to determine whether it is bioequivalent to other cefdinir preparations [3-5]. Cefdinir dispersible tablet disintegrates very fast and disperses homogenously, it is easily to be absorbed, and highly effective. The purpose of this study was to determine the pharmacokinetics and bioequivalence between cefdinir dispersible tablet (test preparation) and cefdinir capsule (reference preparation) and to ascertain the safety in medical practice.

\section{Materials and Methods}

\section{Drugs and reagents}

The test preparation, cefdinir dispersible tablet, was supplied by Tianjin Zhongyang Pharmaceutical Company Ltd.; Batch No.050401 as $100 \mathrm{mg}$ per tablet. The reference preparation, cefdinir capsule, was manufactured by Tianjin Zhongyang Pharmaceutical Company Ltd.; Batch No. 050402 as $100 \mathrm{mg}$ per capsule. The standard substance of cefdinir was supplied by National Institute for the Control of Pharmaceutical and Biological Products, Beijing, People's Republic of China, Batch No.130502-200401, purity $98.3 \%$. Internal standard cefadroxil, purity $95.0 \%$ was supplied by Nationa Institute for the Control of Pharmaceutical and Biological Products, Beijing, People's Republic of China, Batch No.0431-9501. Acetonitrile and methanol were HPLC grade. Potassium dihydrophosphate and phosphoric acid were analytical grade. Double distilled water was used in the preparation of all reagents and mobile phase throughout the study.

\section{Approved procedure}

The study was conducted in accordance with good clinical practice (GCP) guidelines of State Food and Drug Administration in China [6] and The Declaration of Helsinki (as revised in Edinburgh 2000).

The study was approved by Independent Ethical Committee of Tianjin Medical University. Written informed consent was obtained from all subjects after the medical supervisor of the clinical trial explaining the aim, course, and possible risks of the study prior to participation.

\section{Study subjects}

18 healthy Chinese male volunteers were selected in this study. The subjects ranged in age from 20 24 (22 \pm 1$)$ years old, height from

${ }^{*}$ Corresponding author: Prof. Jian-Shi Lou, Department of Pharmacology, Tianjin Medical University, 22 Qi Xiang Tai Road, Tianjin 300070, P.R.China, Tel: 86-22 23542523; E-mail: jianshilou@163.com

Received May 19, 2011; Accepted July 07, 2011; Published July 09, 2011

Citation: Zhang CL, Jiao JJ, Wu YN, Song JQ, Gao WZ, et al. (2011) Study on Pharmacokinetics and Bioequivalence of Cefdinir Dispersible Tablet in Healthy Chinese Volunteers. J Bioequiv Availab 3: 114-117. doi:10.4172/jbb.1000070

Copyright: $\odot 2011$ Zhang CL, et al. This is an open-access article distributed under the terms of the Creative Commons Attribution License, which permits unrestricted use, distribution, and reproduction in any medium, provided the original author and source are credited. 


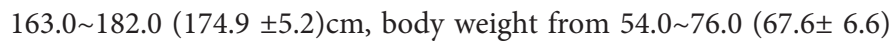
$\mathrm{kg}$, body mass index 20.08 25.06 $(22.05 \pm 1.38) \mathrm{kg} / \mathrm{m}^{2}$. The subjects were selected after completing a thorough medical history, physical examination, laboratory tests (hepatic and renal function tests, blood biochemistry, and urine analysis) and 12 lead electrocardiogram (ECG). The volunteers had no evidence of cardiovascular, pulmonary, hepatic, renal, gastrointestinal, neurologic, endocrine, hematologic disorders or any acute or chronic diseases. Before the study, cephalosporin skin test was performed to the volunteers. They were with negative results. All subjects stopped to use any drugs two weeks before the study and during the study. Cigarette, alcohol and caffeine-containing beverages were forbidden for 48 hours prior the study and during the trial.

\section{Study design}

A single dose, randomized, two-way cross-over study was designed. 18 volunteers were randomly divided into two groups (test and reference). After a 12 hours overnight fasting, all subjects were given a single dosage of cefdinir dispersible tablet 200mg (100mg/ tablet, 2 tablets) or cefdinir capsule $200 \mathrm{mg}$ (100mg/capsule, 2 capsules) orally with $200 \mathrm{ml}$ warm water. $3 \mathrm{ml}$ blood samples were collected in heparinized tubes at the following times on the day of study: immediately prior to drug administration (0h) and at $0.25,0.5,1,1.5$, $2,3,4,6,8,10$ and 12 hours after the drug administration. A cross-over study was followed by 5 days washout period. Regular standardized low-fat meals were provided until 4 hours after dose administration; water intake was allowed after 2 hours.

\section{Safety}

Volunteers' symptoms, signs, vital signs, including blood pressure, heart rate and body temperature were checked in different times after drug administration and ECG, routine laboratory tests were performed before and within a week after the end of drug administration, safety was also monitored by recording reported adverse events.

\section{Drug assay}

For plasma cefdinir concentration assays, analysis was performed with the use of high performance liquid chromatography (HPLC) with ultraviolet assay $[7,8]$. The instrumentation system used in this work consisted of Agilent 1100 HPLC (Agilent Technologies Inc. USA), CAY-1 vortex (Beijing Chang-an Instrument Company), chromatographic column: Kromasil ODS, $250 \times 4.6 \mathrm{~mm}, 5 \mu \mathrm{m}$ (Dalian
Chemical Physics Institute, Academy of Sciences, China), Serial No. C18 B040905.

The mobile phase used was acetonitrile $0.015 \mathrm{M}: \mathrm{KH}_{2} \mathrm{PO}_{4}$ (use phosphoric acid adjust $\mathrm{pH}=3.8)(\mathrm{V} / \mathrm{V} 15: 85)$ at flow rate $1 \mathrm{ml} / \mathrm{min}$; wave length of UV detector: $280 \mathrm{~nm}$. Injection volume was $20 \mu \mathrm{l} .60 \mu \mathrm{l}$ of $31.35 \mu \mathrm{g} / \mathrm{ml}$ internal standard cefadroxil was added to $0.3 \mathrm{ml}$ of plasma sample and then $550 \mu \mathrm{l}$ cold methanol was added to the plasma sample. Vortex for $1 \mathrm{~min}$. After mixing well, centrifuged for $15 \mathrm{~min}$ at 3500rpm. Supernatant was aspirated and transferred into another test tube, then $2 \mathrm{ml}$ of dichloro-methane was added . Votex for $1 \mathrm{~min}$, centrifuged for $10 \mathrm{~min}$ at the speed of $12000 \mathrm{rpm}$. $20 \mu \mathrm{l}$ of the supernatant was injected into HPLC system for analysis.

\section{Pharmacokinetic analysis}

Pharmacokinetic parameters were calculated from plasma concentration-time by non-compartmental methods. The maximum plasma concentration $\left(\mathrm{C}_{\max }\right)$ and time to maximum plasma concentration $\left(\mathrm{T}_{\max }\right)$ were obtained directly from the observed concentration-time profiles. Linear trapezoidal rule was used to estimate the area under the plasma concentration versus time curve (AUC) from 0 to the last measurable concentration $\left(\mathrm{AUC}_{0 . \mathrm{t}}\right)$. The area under the plasma concentration versus time curve from 0 to infinity $\left(\mathrm{AUC}_{0-\infty}\right)$ was calculated as $\mathrm{AUC}_{0-\mathrm{t}}+\mathrm{Ct} / \mathrm{Ke}$, where $\mathrm{Ct}$ was the last measurable concentration. Ke was the elimination rate constant. The terminal elimination half-life $\left(t_{1 / 2}\right)$ was calculated as $0.693 / \mathrm{Ke}$. Bioavailability (F) was calculated according the equation: $\mathrm{F}=\left[\mathrm{AUC}_{0}\right.$. ${ }_{t}$ (test) $/ \mathrm{AUC}_{0-\mathrm{t}}$ (reference) $] \times 100 \%$.

\section{Statistical analysis}

All data were expressed as mean \pm standard deviation (SD), t-test was used as statistical process. $\mathrm{AUC}_{0-\mathrm{t}} \mathrm{AUC}_{0-\infty}, \mathrm{C}_{\max }$ and $\mathrm{t}_{1 / 2}$ were considered as primary variables for the aim of bioequivalence analysis. The bioequivalence of two products were assessed by means of an analysis of variance (ANOVA) for crossover design, paired two one-sided t-test, and calculating standard $90 \%$ confidence intervals of reference drug. The parameter $\mathrm{T}_{\max }$ was analyzed with Wilcoxon's rank sum test. The products were considered bioequivalent, if the difference between two compared parameters was statistically insignificant $(\mathrm{P}>0.05)$ and the mean values of the product fall within $90 \%$ confidence intervals of the reference drug for AUC and $\mathrm{C}_{\max }$, that are the ranges accepted by State Food and Drug Administration of China) [6].All statistical tests
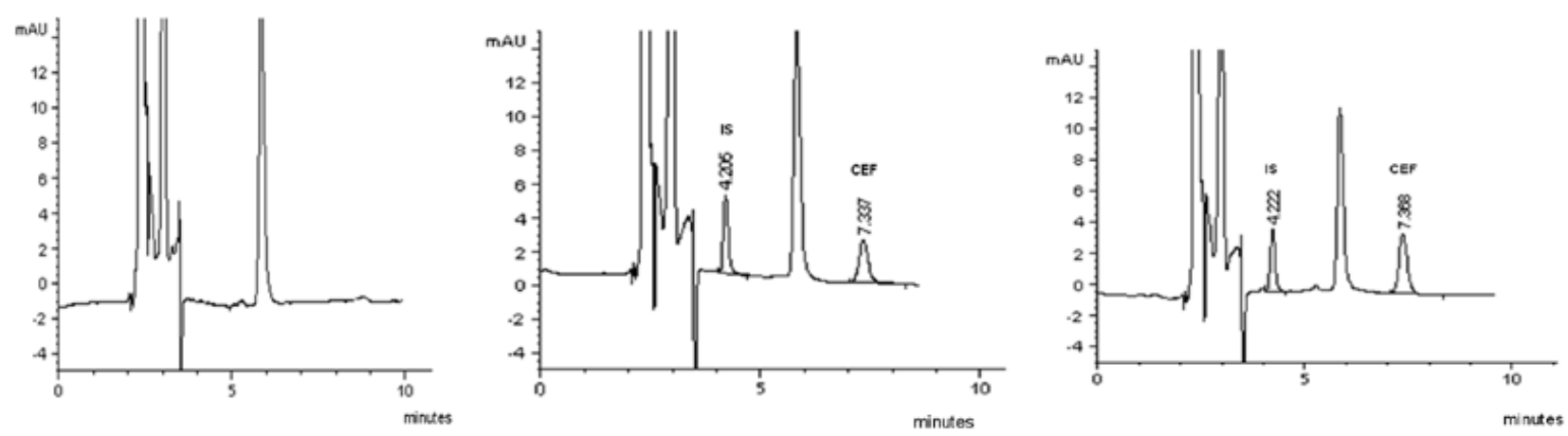

\section{A B C}

Figure 1: A.HPLC chromatogram of blank plasma; B.Blank plasma spiked with cefdinir and internal standard; C. Plasma sample of volunteer after administration of cefdinir, and spiked with internal standard. 
Citation: Zhang CL, Jiao JJ, Wu YN, Song JQ, Gao WZ, et al. (2011) Study on Pharmacokinetics and Bioequivalence of Cefdinir Dispersible Tablet in Healthy Chinese Volunteers. J Bioequiv Availab 3: 114-117. doi:10.4172/jbb.1000070

used a 5\% level of significance to determine significance. All $\mathrm{P}$ values cited were 2-tails.

\section{Results}

\section{Method validity}

The analytical method used for measurement of cefdinir in plasma was proved to be accurate and sensitive. The regression equation was described as $\mathrm{Y}=0.9032 \mathrm{X}+0.0001(\mathrm{r}=1.0016, \mathrm{n}=3)$ ( $\mathrm{Y}$ : peak area ratio of cefdinir and internal standard [S/IS], X:plasma concentration of cefdinir), using $(1 / \mathrm{C})$ weighted least-square regression. The linear relationship was obtained from the peak area between cefdinir (plasma concentration from 0.065 to $8 \mu \mathrm{g} / \mathrm{ml})$ and internal standard $(\mathrm{r}=1.0016)$, the lower limit of quantitation (LOQ) was $0.0625 \mu \mathrm{g} / \mathrm{ml}$. The intra-day and inter-day coefficients of variation $(\mathrm{CV} \%)$ for these three quality control standard $(0.125,1,8 \mu \mathrm{g} / \mathrm{ml})$ were $4.41 \%$ and $6.01 \%$ respectively. The total precision was $5.21 \%$. The intra-day and inter-day accuracy for three quality control standard $(0.125,1,8 \mu \mathrm{g} / \mathrm{ml})$ were $105.48 \pm 1.98 \%$ and $106.90 \pm 6.48 \%$ respectively. Total accuracy was $106.19 \pm 5.57 \%$. The mean absolute recovery was $95.38 \pm 4.27 \%$. The retention time for cefdinir and internal standard were $7.4 \mathrm{~min}$ and $4.2 \mathrm{~min}$ separately.

The representative HPLC chromatograms of cefdinir in plasma are shown in Figure 1.

\section{Pharmacokinetics, bioavailability and bioequivalence}

The mean plasma concentration-time curves after oral administration of $200 \mathrm{mg}$ cefdinir in 18 healthy Chinese volunteers are shown in Figure 2, and the main pharmacokinetic parameters of cefdinir dispersible tablet (test) and cefdinir capsule (reference) were listed in Table 1.

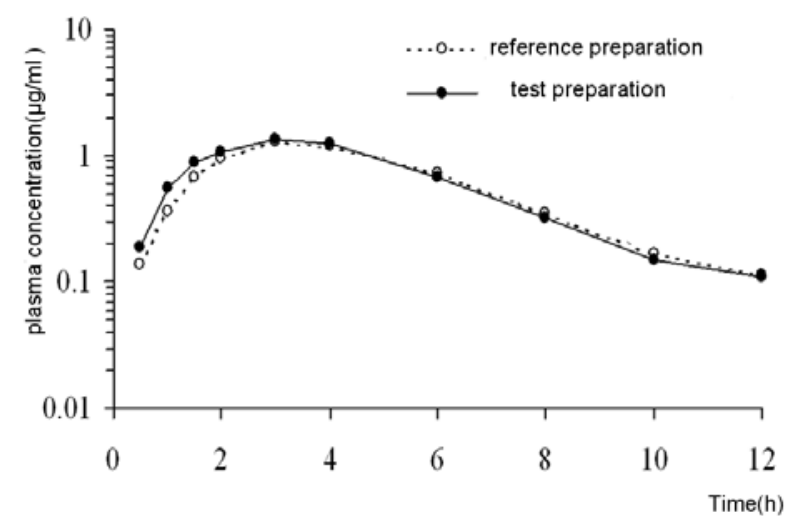

Figure 2: Mean plasma concentration-time curves of cefdinir after a single oral administration of $200 \mathrm{mg}$ cefdinir test and reference preparations in healthy Chinese volunteers $(n=18$, mean $\pm S D)$.

\begin{tabular}{|l|l|l|}
\hline Parameters & Test preparation & Reference preparation \\
\hline Cmax $(\mu \mathrm{g} / \mathrm{ml})$ & $1.52 \pm 0.48$ & $1.42 \pm 0.39$ \\
\hline Tmax $(\mathrm{h})$ & $3.08 \pm 0.73$ & $3.22 \pm 0.81$ \\
\hline $\mathrm{t}_{1 / 2}(\mathrm{~h})$ & $2.04 \pm 0.53$ & $1.87 \pm 0.29$ \\
\hline $\mathrm{AUC}_{0-\mathrm{t}}\left(\mu \mathrm{g} / \mathrm{ml}^{\left.-\mathrm{h}^{-1}\right)}\right.$ & $7.12 \pm 1.85$ & $6.86 \pm 1.60$ \\
\hline $\mathrm{AUCO}_{0-\infty}\left(\mu \mathrm{g} / \mathrm{ml}^{-1} \mathrm{~h}^{-1}\right)$ & $7.67 \pm 2.01$ & $7.38 \pm 1.85$ \\
\hline $\mathrm{F}_{0-\mathrm{t}}(\%)$ & $103.53 \pm 11.50$ & \\
\hline $\mathrm{F}_{0-\infty}(\%)$ & $104.13 \pm 11.83$ & \\
\hline
\end{tabular}

Table 1: Main pharmacokinetic parameters of cefdinir after a single oral dose of $200 \mathrm{mg}$ cefdinir test and reference preparations in healthy Chinese male volunteers $(n=18$, mean $\pm S D)$.

\begin{tabular}{|l|l|l|l|l|l|l|}
\hline Author & $\begin{array}{l}\text { Dose } \\
(\mathrm{mg})\end{array}$ & $\begin{array}{l}\mathrm{Tmax} \\
(\mathrm{h})\end{array}$ & $\begin{array}{l}\text { Cmax } \\
(\mu \mathrm{g} / \mathrm{ml})\end{array}$ & $\begin{array}{l}\mathrm{t}_{1 / 2} \\
(\mathrm{~h})\end{array}$ & $\begin{array}{l}\mathrm{AUC}_{0-\mathrm{t}} \\
\left(\mu \mathrm{g} / \mathrm{ml}^{-1} \mathrm{~h}^{-1}\right)\end{array}$ & $\begin{array}{l}\mathrm{F} \\
(\%)\end{array}$ \\
\hline This study & 200 & $3.08 \pm 0.30$ & $1.52 \pm 0.48$ & $2.04 \pm 0.53$ & $7.17 \pm 1.85$ & $103.53 \pm 11.50$ \\
\hline $\begin{array}{l}\text { Zhang } \\
\text { XinPing }\end{array}$ & 400 & $3.48 \pm 0.53$ & $2.10 \pm 0.32$ & $2.41 \pm 0.39$ & $9.51 \pm 1.65$ & $96.03 \pm 14.80$ \\
\hline Ren Ping $^{[4]}$ & 100 & $3.375 \pm 0.940$ & $0.920 \pm 0.236$ & $1.826 \pm 0.6$ & $4.305 \pm 1.08$ & $101.0 \pm 23.2$ \\
\hline $\begin{array}{l}\text { Wang } \\
\text { Feng }\end{array}$ & 200 & $3.10 \pm 0.86$ & $1.34 \pm 0.44$ & $1.55 \pm 0.22$ & $6.45 \pm 2.01$ & $100.08 \pm 15.30$ \\
\hline
\end{tabular}

Table 2: Comparison of the pharamacokinetic parameters of cefnidir.

The statistic analysis showed that there were no significant difference for pharmacokinetic parameters $C_{\max }, T_{\max }, t_{1 / 2}, A U C_{0-t^{\prime}}$ $\mathrm{AUCO}_{0-\infty}$ between two preparations $(\mathrm{P}>0.05)$. The $90 \%$ confidential interval of $\mathrm{AUC}_{0-\mathrm{t}}, \mathrm{AUCO}_{0-\infty}, \mathrm{C}_{\max }$ of reference drug were 6.28 7.78 $\mu \mathrm{g} /$ $\mathrm{ml} \bullet \mathrm{h}^{-1}, 6.82 \sim 8.30 \mu \mathrm{g} / \mathrm{ml}^{\circ} \mathrm{h}^{-1}$ and $1.19 \sim 1.84 \mu \mathrm{g} / \mathrm{ml}$ respectively. The $90 \%$ confidential interval of the $\mathrm{AUC}$ and $\mathrm{C}_{\max }$ were within the range of $80 \% \sim 125 \%$ and $70 \% \sim 143 \%$ respectively. The means of $\mathrm{AUC}_{0 \mathrm{t}}, \mathrm{AUC}_{0-\infty}$ and $\mathrm{C}_{\max }$ for the test drug were all within the $90 \%$ CIs of reference drug. According to the bioequivalence criteria, these two preparations were bioequivalent [6].

\section{Safety evaluation}

Test and reference preparations were well tolerated at the dose administered by all volunteers. No adverse events occurred in this study. During the study none of the changes in laboratory test values and vital signs were considered clinically important and the biochemical parameters were within the reference range. There were no dropouts for the volunteers.

\section{Discussion}

A single oral dose of $200 \mathrm{mg}$ cefdinir dispersible tablet and cefdinir capsule were given to 18 healthy Chinese male volunteers in randomized, two-treatment, two-period crossover study. The plasma drug concentrations were determined by the validated HPLC with UV assay. It was a specific, sensitive and reproducible procedure and a suitible, valuable tool in the assessment of pharmacokinetics and bioavailability of cefdinir dispersible tablet. The present pharmacokinetic study indicated that both preparations were readily absorbed from gastrointestinal tract with a Tmax of approximately 3 hours. The mean concentration-time profiles of two preparations were closely similar (Figure 2).

The pharmacokinetic parameters of two preparations in this study were similar to that of other reports [3-5], Compare the pharmacokinetic parameters of other reports with this study, the results were shown in Table 2 .

Their $\mathrm{T}_{\max }, \mathrm{t}_{1 / 2}$ were similar. $\mathrm{C}_{\max }$ of same dosage of cefdinir (200mg) were similar, $\mathrm{C}_{\max }$ of $400 \mathrm{mg}$ cefdinir dispersible tablet was larger than that of $200 \mathrm{mg}$ and $100 \mathrm{mg}$ cefdinir dispersible tablet. $\mathrm{AUC}_{0-\mathrm{t}}$ of same dosage of cefdinir $(200 \mathrm{mg})$ were similar. $\mathrm{AUC}_{0-\mathrm{t}}$ of $400 \mathrm{mg}$ cefdinir dispersible tablet was larger than that of $200 \mathrm{mg}, 100 \mathrm{mg}$ cefdinir dispersible tablet. Parameters of cefdinir in healthy volunteers are different from that of hemodialysis patients [9]. The dosage for renal failure patients was $100 \mathrm{mg}$ and the dosage for healthy volunteers in this study was $200 \mathrm{mg}$. The mean $t_{1 / 2}$ during hemodialysis for the patients was significantly less $(2.76 \pm 1.01 \mathrm{~h})$ than that in the test without dialysis $(16.95 \pm 1.20 \mathrm{~h}) . \mathrm{t}_{1 / 2}$ for healthy subjects was shorter $(2.04 \pm 0.53 \mathrm{~h})$ than that of hemodialysis patients $(2.76 \pm 1.01 \mathrm{~h}) . \mathrm{AUC}_{0-\infty}$ in the test with dialysis was $43 \%\left(30.18 \pm 12.03 \mu \mathrm{g} / \mathrm{ml}^{\circ} \bullet^{-1}\right)$ of that in the test without 
Citation: Zhang CL, Jiao JJ, Wu YN, Song JQ, Gao WZ, et al. (2011) Study on Pharmacokinetics and Bioequivalence of Cefdinir Dispersible Tablet in Healthy Chinese Volunteers. J Bioequiv Availab 3: 114-117. doi:10.4172/jbb.1000070

hemodialysis. It was still larger than that of healthy volunteers $(7.67 \pm$ $2.01 \mu \mathrm{g} / \mathrm{ml} \mathrm{h}^{-1}$ ). For the safety of the volunteers, cephalosporin skin testing has been undertaken before volunteers were selected to enroll in this trial. The positive and negative predictive values of the results are less well established than those of penicillin [10]. Throughout the whole study period, there were no adverse events reported.

In the study, $\mathrm{C}_{\max }, \mathrm{AUC}_{0-\mathrm{t}}, \mathrm{AUCO}_{0-\infty}$ of two preparations did not differ suggesting the plasma profiles generated by the test preparation were comparable to those of the reference preparation. ANOVA, after logtranformation of the data, showed no statistically significant difference between two preparations $(\mathrm{P}>0.05)$. From the result of bioequivalence analysis, the parametric mean values of the pharmacokinetic characteristics for test drug were within the bioequivalence acceptable ranges of $80 \sim 125 \%$ and $70 \sim 143 \%$ respectively for AUC and $C_{\max }$. These results were confirmed by Schuirmann's two one-sided $t$ tests, which indicated that the lower and upper limits of the calculated $t$ value were greater than the critical t value for $\mathrm{AUC}_{0-\mathrm{t}}, \mathrm{AUCO}_{0-\infty}$ and $\mathrm{C}_{\max }$. Therefore, two cefdinir preparations can be considered bioequivalent.

$\mathrm{PK} / \mathrm{PD}$ research for antimicrobial agents is very important and it is same for cefdinir. If we want to evaluate the pharmacologic effects of cefdinir, it is necessary to perform the pharmacodynamics and pharmacokinetics of this drug. The important $\mathrm{PK} / \mathrm{PD}$ parameters for cefdinir are time over MIC and AUC/MIC, especially time over MIC, because this drug is time-dependent, but it is not necessary to supply $\mathrm{PK} / \mathrm{PD}$ parameters for cefdinir BE application in China.

In conclusion, cefdinir dispersible tablet and cefdinir capsule are bioequivalent, and these two preparations can be believed to have same therapeutic effect in medical practice.

\section{References}

1. Perry CM, Scott LJ (2004) Cefdinir: A review of Its Use in Management of Mild and Moderate Bacterial Infections. Drug 64: 1433-64.

2. Guay DR (2000) Cefdinir: An Expanded-Spectrum Oral Cephalosporin. Ann Pharmacother 34: 469-77.

3. Zhang X, Zhao L, Lei G (2007) Bioequivalence of Cefdinir Dispersible Tablet in Healthy Volunteers. China Pharmacy 18: 2514-2516.

4. Ping R, Gang Z, Xu K, Shi-Fen G, Hui C (2009) Study on Human Pharmacokinetics and Bioequivalence of Cefdinir Dispersible Tablet. Herald of Medicine 28: 168-170.

5. Wang Feng, Li Xiang-Hong, Sun Hua. (2010) Study on Bioequivalence of Domestic Cefdinir Dispersible Tablet. Pharmacy Today 20: 22-24,45.

6. Guidance for bioavailability and bioequivalence studies for chemical drug products. China: State Food and Drug Administration, March 2005.

7. Xing-Lin W (2003) Determination of cefdinir and its related substances by high performance liquid chromatography. Chinese Journal of New Drug 12: 114117.

8. Okamoto Y, Itoh K, Namiki Y, Matsushita J, Fujioha M, et al. (1996) Method development for determination of cefdinir and its related substances by high performance liquid chromatography. J Pharm Biomed Anal 14: 739-748.

9. Hishida A, Ohishi K, Nagashima S, Kanamaru M, Obaca M, et al. (1998) Pharmacokinetic study of an oral cephalosporin, cefdinir, in hemodialysis patients. Antimicrob Agents Chemother 42: 1718-1721.

10. Pichichero ME (2005) A Review of Evidence Supporting the American Academy of Pediatrics Recommandation for Prescribing Cephalosporin Antibiotic for penicillin Allergic Patients. Pediatrics 115: 1048-1057. 